\title{
Discapacidad visual no corregible con anteojos
}

\author{
Visual impairment not correctable with glasses
}

Ko F y col. Jama 2012; 308 : 2361-2368

\section{Objetivo}

Estimar la prevalencia de discapacidad visual no refractiva y describir su relación con factores de riesgo demográficos y sistémicos, incluyendo el diagnóstico de diabetes.

\section{Diseño}

Este estudio analizó datos provenientes del National Health and Nutrition Examination Survey (NHANES), una muestra de tipo transversal de población no institucionalizadas de Estados Unidos a cuyos integrantes se les realizaron estudios para determinar su estado de salud en dos períodos: 1999 a 2002 y 2005 a 2008.

\section{Lugar}

Estados Unidos.

\section{Pacientes}

Mayores de 20 años que hubieran completado sus controles de salud. Los datos demográficos (edad, sexo, raza, años de escolaridad, ingresos y cobertura de salud) fueron recolectados a través de encuestas en los hogares, así como el estatus tabáquico y el diagnóstico de diabetes. Fueron clasificados como portadores de una discapacidad visual quienes tuvieron un registro de agudeza visual corregida menor a 20/40 en el ojo de mejor visión.

\section{Resultados}

La prevalencia de discapacidad visual durante el primer período fue de $1,4 \%$ y en el segundo, $1,7 \%(p=0,03)$. En ambos períodos los factores que mostraron asociación independiente a la discapacidad visual fueron la falta de cobertura médica, la baja educación, la pobreza y el diagnóstico de más de diez años de diabetes, cuya prevalencia se incrementó globalmente un $22 \%$, desde 2,8 a 3,6\% ( $p=0,02)$; y particularmente entre los blancos no hispanos de 20 a 39 años, desde $0,3 \%$ a $0,7 \%$ $(p<0.001)$.

\section{Conclusión}

La prevalencia de discapacidad visual no refractiva fue significativamente mayor en el segundo período, y podría ser atribuible a una mayor prevalencia de diabetes de diez o más años de evolución.

Fuente de financiamiento: Centro de Control de Enfermedades, Instituto Nacional de la Salud y la Nutrición de EE.UU. Conflictos de interés: El Dr. Friedman es Consultor de Alcon, Bausch and Lomb, Merck, Pfizer, Allergan, Nidek y QLT; y el Dr. Ko recibió apoyo para viajes a congresos para el estudio y otros fines del National Eye Institute, así como becas por asistencia de redacción, medicinas, equipamiento o soporte administrativo.

\section{Comentario}

Luego de analizar los dos períodos se encontró que la discapacidad visual no refractiva aumentó un $21 \%$, y luego de ajustar por potenciales confundidores mostró asociación estadísticamente significativa con la prevalencia de diabetes de más de diez años de evolución, que se incrementó entre los dos períodos y que mostró un efecto de tipo "dosisrespuesta", lo que daría mayor fuerza a algún tipo de hipótesis causal.

Si la tendencia observada en este estudio se mantiene en el tiempo el número de pacientes jóvenes con discapacidad visual no refractiva irá en aumento con el consecuente com- promiso de su calidad de vida, su productividad y el consiguiente aumento de los costos en salud.

\section{Conclusiones del comentador}

La diabetes está considerada como la causa más frecuente de ceguera no reversible en los países industrializados ${ }^{1-2}$.

El presente estudio documenta la tendencia de un aumento en el diagnóstico de diabetes sobre todo en personas jóvenes económicamente productivas ${ }^{3}$. Al menos parte de esta tendencia estaría vinculada a un aumento en la prevalencia de obesidad en adultos jóvenes asociada a la industrialización.

Tomás Cubero [ Servicio de Oftalmología del Hospital Italiano de Buenos Aires. tomas.cubero@ hospitalitaliano.org.ar ]

Cubero T. Discapacidad visual no corregible con anteojos. Evid Act Pract Ambul Abr-Jun 2013;16(2):51. Comentado de: Ko F y col. Prevalence of Nonrefractive Visual Impairment in US Adults and Associated Risk Factors, 1999-2002 and 2005-2008. JAMA. 2012;308(22):2361-2368. PMID: 23232894

\section{Referencias}

1. Zachary T, Bloomgarden, M. Diabetic Retinopathy. Diabetes Care 31: 1080-1083, 2008

2. Sociedad española de retina y vítreo. Manejo de las complicaciones oculares de la diabetes. Retinopatía diabética y edema macular. Madrid 2009

3. Early Treatment Diabetic Retinopathy Study Research Group. Grading diabetic retinopathy from stereoscopic color fundus photographs an extension of the modified Airlie House classification. ETDRS Report number 10. Ophthalmology 1991; 98: 786-806 